\title{
Cardiovascular and coordination training differentially improve cognitive performance and neural processing in older adults
}

\author{
Claudia Voelcker-Rehage* , Ben Godde and Ursula M. Staudinger \\ Jacobs Center on Lifelong Learning and Institutional Development, Jacobs University Bremen, Bremen, Germany
}

Edited by:

Hauke R. Heekeren, Max Planck Institute for Human Development, Germany

Reviewed by:

Lars Nyberg, Umeå University, Sweden

Patricia Reuter-Lorenz, University of Michigan, USA

${ }^{*}$ Correspondence:

Claudia Voelcker-Rehage, Jacobs Center on Lifelong Learning and Institutional Development, Jacobs University Bremen, Campus Ring 1, 28759 Bremen, Germany.

e-mail: c.voelcker-rehage@jacobsuniversity.de

\begin{abstract}
Recent studies revealed a positive influence of physical activity on cognitive functioning in older adults. Studies that investigate the behavioral and neurophysiological effects of type and long term duration of physical training, however, are missing. We performed a 12-month longitudinal study to investigate the effects of cardiovascular and coordination training (control group: relaxation and stretching) on cognitive functions (executive control and perceptual speed) in older adults. We analyzed data of 44 participants aged 62-79 years. Participants were trained three times a week for 12 months. Their physical and cognitive performance was tested prior to training, and after 6 and 12 months. Changes in brain activation patterns were investigated using functional MRI. On the behavioral level, both experimental groups improved in executive functioning and perceptual speed but with differential effects on speed and accuracy. In line with the behavioral findings, neurophysiological results for executive control also revealed changes (increases and reductions) in brain activity for both interventions in frontal, parietal, and sensorimotor cortical areas. In contrast to the behavioral findings, neurophysiological changes were linear without indication of a plateau. In both intervention groups, prefrontal areas showed decreased activation after 6 and 12 months when performing an executive control task, as compared to the control group, indicating more efficient information processing. Furthermore, cardiovascular training was associated with an increased activation of the sensorimotor network, whereas coordination training was associated with increased activation in the visual-spatial network. Our data suggest that besides cardiovascular training also other types of physical activity improve cognition of older adults. The mechanisms, however, that underlie the performance changes seem to differ depending on the intervention.
\end{abstract}

Keywords: physical training, cognition, older adults, brain aging, functional MRI, fitness

\section{INTRODUCTION}

The importance of physical activity for the improvement and preservation of cognitive abilities in healthy older people has repeatedly been shown during recent decades (e.g., Colcombe and Kramer, 2003; Lustig et al., 2009). The usual target age of the studies in later adulthood reflects age-related decline in cognitive performance, such as a diminished speed of information processing or a lower capacity of working memory (Cabeza, 2001; Park and Reuter-Lorenz, 2009; Bäckman et al., 2010; Lövdén et al., 2010). Colcombe et al. (2004) provided first neurophysiological evidence that after cardiovascular training cognitive resources were applied more effectively and/or more flexibly. Older adults participating in a 6-month cardiovascular training intervention showed greater activations in cortical regions associated with the effective control of attention (right middle frontal gyrus, MFG; superior frontal gyrus, SFG; superior parietal lobus, SPL) as well as better inhibition of distracting information as indicated by lower activity in the anterior cingular cortex (ACC). Besides replicating earlier findings, the present study aimed to extend this research by investigating the possibility of differential effects of different types of physical training, and by expanding the intervention interval up to 12 months in order to test whether neurophysiological and/or performance changes continue or level off.
To date, human studies reflect a preference for investigating the effects of cardiovascular training (Kramer and Erickson, 2007). Animal studies, however, have revealed that different kinds of activity might influence the brain metabolism in different ways (Black et al., 1990; Anderson et al., 1994). Cardiovascular training has been found, for example, to increase the density of the capillaries (angiogenesis; Black et al., 1990), the building of new neurons (neurogenesis) or the plasticity of neurotransmitter systems (van Praag et al., 2005), whereas coordinative tasks (such as traversing ropes, crossing obstacles) support sprouting and restructuring of synapses (synaptogenesis; Black et al., 1990). Similarly, our crosssectional data demonstrated that for older adults not only physical fitness indexed by cardiovascular fitness and muscular strength, but also motor fitness including movement speed, balance, coordination, and flexibility showed a strong positive association with cognitive functioning (Voelcker-Rehage et al., 2010). Furthermore, functional brain imaging data of this study revealed that physical and motor fitness differentially related to cognitive processes. Whereas, both physical and motor fitness were related to more efficient cognitive processing, as indicated by less cortical activations in areas responsible for executive control (superior and middle frontal cortex), motor fitness was also related to greater activation 
of the right inferior frontal-posterior parietal network involved in visuo-spatial processing and action initiation (De Graaf et al., 2009). Supportive evidence from behavioral studies is also available from younger populations. In a sample of patients (23-53 years of age) with mild traumatic brain injury Geurts et al. (1999) found an association between performance in static and dynamic balance with performance in the Digit-Symbol Test (a test of working memory and visuo-motor processing). A behavioral cross-sectional study with preschool children also revealed a positive relationship between coordinative (coordination and speed of movement) and cognitive abilities (Razkol Test; Planinsec, 2002). A first intervention study with grade 5 students showed that a 6-week bimanual coordination program improved the reading comprehension skills compared to controls (Uhrich and Swalm, 2007).

We conducted a longitudinal study using two types of training, namely cardiovascular and coordination training. Coordination training was designed as a program to improve fine and grossmotor body coordination such as balance, eye-hand coordination, leg-arm coordination as well as spatial orientation and reaction to moving objects/persons. A third group participated in a stretching and relaxation program serving as a control group. On the behavioral level, we hypothesized a positive effect of both cardiovascular and coordination training on cognitive functioning. On the neurophysiological level, we expected more efficient cognitive processing in areas responsible for executive control due to the participation in the cardiovascular and coordination training program. Previous cross-sectional results revealed a positive relationship between activations in parietal and inferior frontal cortices with motor fitness status (Voelcker-Rehage et al., 2010). Thus, we also expected improved processing in areas involved in visual-spatial processing due to the participation in the coordination training program.

The few studies that have investigated the long term slopes (>6 months) of physical activity interventions on cognitive performance have yield mixed results (Rikli and Edwards, 1991; Smiley-Oyen et al., 2008; Williamson et al., 2009). Smiley-Oyen et al. (2008) demonstrated that for older adults, aged 56-79 years, 10 months of cardiovascular training, but not strength-andflexibility training, had positive effects on demanding executive control tasks (e.g., Stroop Word-Color Task); increases were particularly strong within the first 5 months of the study. Rikli and Edwards (1991) performed a 3-year intervention study (measurement points: at the beginning of the study, after 1 year, and after 3 years) to investigate the effect of a combined training program (endurance, strength, balance, coordination, flexibility) on simple and choice reaction time tasks in older adults (57-85 years) with a positive effect on cognitive processing speed (control group: nonexercise hobby classes). They observed significant improvements for the experimental group within the first year of the study, but no change from year 1 to year 3. Also, Williamson et al. (2009) examined older adults (70-89 years) by using a 12-month combined training program (endurance, strength, balance, flexibility). They found an association between changes in motor and cognitive functioning, but no strong difference between experimental and control group (health education) in cognitive performance (measurement points: at the beginning and end of the study). No study, however, has investigated behavioral and neurophysiological correlates of cognitive changes after a 12 -month training period. It is yet to be determined whether, and if so, when the limits of plasticity are reached on both, the behavioral and neurophysiological level. That is, do neurophysiological changes and cognitive improvement continue throughout a longer training period or not. Due to the scarcity of extant studies covering long intervention periods this part of our study is foremost exploratory. Based on prior behavioral results (Smiley-Oyen et al., 2008), we hypothesized that there was a high likelihood that performance increases would level off after 6 months of training.

In sum, the present study had three aims: (1) to replicate existing results on the effects of shorter term cardiovascular training on cognitive functioning in older adults, (2) to extend these earlier findings by analyzing effects of a longer training period covering 1 year, and furthermore (3) to investigate specific and general effects of cardiovascular training as compared to other training programs, that is, coordination training.

\section{MATERIALS AND METHODS PARTICIPANTS}

Ninety-one participants from the Bremen (Germany) area were included in this study and were randomly assigned to two experimental groups and a control group with respect to their place of residence. All adults participated voluntarily in the study; they were recruited through the member registry of a German health insurance company (DAK) or through advertisements. Participants provided written informed consent to the procedures of the study approved by the ethics committee of the German Psychological Society (DGPs), and received compensation for their travel expenses at the end of the 1-year study amounting to Euro 100. The study conformed to the Code of Ethics of the World Medical Association (Declaration of Helsinki).

Participants had medical clearance and were screened for health restrictions before inclusion in the study by means of a telephone interview. Participants were excluded from the study if they had a history of cardiovascular diseases, any neurological disorder (e.g., self-report of neurological diseases such as a brain tumor, Parkinson's disease, stroke), any other motor or cognitive restrictions (a score of less then 27 in the Mini Mental Status Examination, MMSE; Folstein et al., 1975), or metal devices in the body. Participants who were absent for more than one test day or more then $25 \%$ of the training sessions (calculated independently for each half year of the study) were excluded from data analysis. The remaining group consisted of 44 participants between 63 and 79 years ( 28 women and 16 men, $M=69.64$, $\mathrm{SD}=3.84$; cardiovascular training $N=17,12$ women, 5 men; coordination training: $N=16,10$ women, 6 men; control group: $N=11,6$ women, 5 men; cf. Table 1 for sample characteristics within each training group).

\section{SCREENING}

All participants were given a demographics and health questionnaire supplemented by personal interviews to determine the characteristics of the sample and self-reported health status. No participant had to be excluded from the study due to his or her health status. Participants of the experimental and the control groups were statistically similar to one another on measures of age, years of formal education, IQ, health, activity index, body 
Table 1 | Demographic information for participants of the two experimental groups (cardiovascular and coordination training) and the control group.

\begin{tabular}{|c|c|c|c|c|c|c|}
\hline Characteristic & \multicolumn{2}{|c|}{ Cardiovascular training } & \multicolumn{2}{|c|}{ Coordination training } & \multicolumn{2}{|c|}{ Control group } \\
\hline Education & 13.00 & 2.96 & 12.06 & 3.64 & 12.09 & 2.34 \\
\hline 10 & 51.42 & 5.77 & 48.40 & 5.66 & 49.93 & 5.91 \\
\hline Health & 1.35 & 0.93 & 1.31 & 1.14 & 1.36 & 1.75 \\
\hline BMI & 27.44 & 4.31 & 25.88 & 2.60 & 26.25 & 3.43 \\
\hline Hypertension & 0.12 & 0.33 & 0.50 & 0.52 & 0.18 & 0.41 \\
\hline ERT & 0.24 & 0.44 & 0.33 & 0.49 & 0.09 & 0.31 \\
\hline Positive affect & 3.74 & 0.67 & 3.68 & 0.53 & 3.76 & 0.54 \\
\hline
\end{tabular}

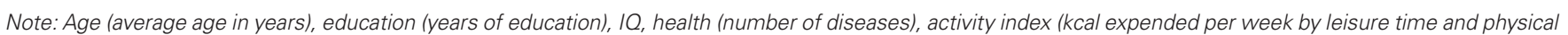

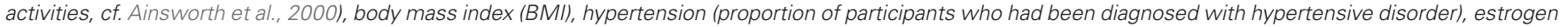

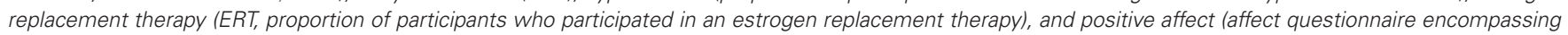
high and low arousal, Kessler and Staudinger, 2009). There was no significant group effect for any of the measures.

mass index (BMI), hypertension, estrogen replacement therapy (for women only), and positive affect [always $F(2,42)<2.75$, $p>0.08$; cf. Table 1].

IQ was measured with a test battery from the Berlin aging study (cf. Li et al., 2004). Seven tests were used reflecting five primary intellectual abilities: (a) Perceptual speed, measured by the mean performance of Digit-Symbol Substitution and Identical Picture Tests, (b) reasoning, measured by the mean performance of tests of Figural Analogies and Letter Series, (c) memory, measured by Paired Associate Test, (d) verbal fluency, measured by the test of naming names of animals, and (e) verbal knowledge, measured by Vocabulary Test. Performance scores were transformed into $T$ scores $(M=50, \mathrm{SD}=10)$ and a mean IQ-score was calculated.

All participants were screened for normal vision (Freiburg Visual Acuity Test; Bach, 2007) and hearing (simultaneous auditory thresholds at multiple frequencies and for both ears; presentation software: Neurobehavioral Systems, Albany, Canada; Yund, 2003). No participant was excluded based on his or her visual or hearing test.

\section{COGNITIVE TASKS}

Two cognitive tests were applied reflecting perceptual speed and executive control.

\section{Executive control}

Executive control was measured by a modified version of the Flanker Task with three response conditions (Li et al., 2004). Participants were asked to identify a colored target in the center (red or green) surrounded by four distractors by button press. In the congruent condition, the color of the distractors was the same as the color of the target, whereas in the incongruent condition, the color of the distractors was identical to the color of the stimulus that required the competing response. In the neutral condition, the color of the distractors (blue) differed both from the color of the target and the color of the stimulus that required the competing response. To separate the pure motor response from executive control processes, in the control condition white targets with white distractors were used and participants were asked to press any button (Voelcker-Rehage et al., 2010). Participants underwent six 30-s blocks of flanker and two 30-s blocks of control trials presented in a randomized order (fixation cross exposure time $300 \mathrm{~ms}$, intermediate blank period $200 \mathrm{~ms}$, stimulus duration $300 \mathrm{~ms}$, reaction period until a response was made or $1000 \mathrm{~ms}$ has passed, mean random trial variance $150 \mathrm{~ms}$, inter-block break duration $30 \mathrm{~s}$ ). All trials within each test block were of the same type (congruent, incongruent, neutral, control). Participants performed one practice block prior to testing to ensure that they understood the directions for the task.

\section{Perceptual speed}

Perceptual speed was measured by a Visual Search Task (Hommel et al., 2004). The stimuli used in the Visual Search Task were filled and unfilled squares and circles (Hommel et al., 2004). In feature search, participants were asked to search for a filled circle (target) among 2, 8, or 14 empty circles (distractors), respectively. In conjunction search, participants were asked to search for the target among 2, 8, or 14 unfilled circles, respectively, and filled squares (distractors). An area of $18.5 \times 14 \mathrm{~cm}$ of the screen was used to display the objects. This area was further split into four invisible inner and four outer regions. During a block of trials, the target randomly appeared for an equal amount of time in each of the eight regions; the distractors appeared randomly at various positions in either region. Each participant performed four blocks of 48 trails each in a randomized order with the possible combinations of three display sizes (2, 8, or 14 items), two conditions (target present or absent), and eight target locations (four inner and four outer quadrants, which mattered in target-present conditions only; fixation cross exposure time $300 \mathrm{~ms}$, intermediate blank period $200 \mathrm{~ms}$, stimulus duration until a response was made or $5000 \mathrm{~ms}$ has passed, inter-block break duration $5000 \mathrm{~ms}$ ). Participants indicated the presence of the target by pressing one key (right) as quickly and accurately as possible, and they indicated the absence of a target by pressing the left key. Participants did one practice block prior to testing to ensure that they understood the directions for the task. 


\section{FITNESS ASSESSMENT}

Fitness was assessed by using a battery of six tests representing cardiovascular and motor fitness, respectively.

\section{Assessment of cardiovascular fitness}

Cardiovascular fitness was assessed by spiroergometry (ZAN300, measurement system for oxygen consumption and indirect calorimetry assessment). The assessment of cardiovascular fitness served to calculate the individual training heart rate (HR) for the cardiovascular training, and in the sense of a manipulation check to assess the physical training effects, that is, cardiovascular training should improve cardiovascular fitness, whereas coordination training and activities of the control group should not. During spiroergometry participants completed a submaximal graded exercise test using a ramp-like treadmill protocol (modified Porszasz et al. (2003) protocol) on a Lode Valiant motor-driven treadmill with electrocardiography activity monitored by a 10-lead fully digital stress system (Kiss, GE Medical Systems) as reported elsewhere (Voelcker-Rehage et al., 2010). For training prescriptions (cardiovascular group) we calculated the aerobic $\left(\mathrm{AerT}_{\mathrm{GE}}\right)$ and anaerobic $\left(\mathrm{AnT}_{\mathrm{GE}}\right)$ gas exchange thresholds. The Aer $\mathrm{T}_{\mathrm{GE}}$ of healthy, untrained older people occurred at $\sim 60 \% \mathrm{VO}_{2}$ peak (Prioux et al., 2000). For data analysis the mean $\mathrm{VO}_{2}$ of the highest complete performance level achieved by the participants was used as reported elsewhere (cf. Voelcker-Rehage et al., 2010).

\section{Assessment of motor fitness}

Tests of motor fitness were feet tapping (Voelcker-Rehage and Wiertz, 2003) as indicator of action speed, a stick-fall-test (Ehrler and Huth, 2000) as indicator of reaction speed, and one-leg-stand with eyes open and closed (Ekdahl et al., 1989) as indicators of balance. The feet tapping test required participants to tap with both feet simultaneously in a sitting position across a marker at the floor in front of them (number of taps within $20 \mathrm{~s}$ were counted, the best of two trials was selected for analysis). In the stick-fall-test, participants were asked to grip a falling stick as soon as possible with their dominant and nondominant hand (three trials with right and left hand, falling distance in $\mathrm{cm}$ was measured, mean of the best trial of the right and left hand was used for data analysis). In the one-leg-stand participants were asked to stand on one leg with the other slightly flexed and looking straight ahead. Compensatory movement of arms and the lifted leg but not of the standing leg were accepted. Participants performed three trials standing on the right and left leg each. Duration of standing in seconds (maximum 20 s) was noted for each trial and the mean of the best trail with the right and left leg was used for data analysis. The one-leg-stand with eyes closed was performed accordingly.

\section{INTERVENTIONS}

Participants took part in one of three intervention groups, three times a week, and $1 \mathrm{~h}$ each for 12 months.

\section{Cardiovascular training}

Participants of the cardiovascular training group participated in a walking intervention designed to improve cardiorespiratory fitness (aerobic endurance). Training intensity prescriptions were based on HR responses to spiroergometry exercise testing. Participants of the cardiovascular training group (walking training) trained with their individual HR in an intensity zone at and above Aer $_{\mathrm{GE}}$ (but below
$\mathrm{AnT}_{\mathrm{GE}}$; extensive training; Meyer et al., 2005). Intensity levels began at AerT $\mathrm{T}_{\mathrm{GE}}$ and increased to above $\mathrm{Aer}_{\mathrm{GE}}$ over the course of the trial. The duration of the walking training began at 35 min per session and increased until $45-50$ min per session after $\sim 3$ months. This duration was maintained for the remaining 9 months of the study. Intensity levels were monitored at each session by HR assessment, exercise duration, and subjective judgment of training intensity. Values and details of training sessions (mean training HR, training duration, blood pressure, and feelings) were recorded in an exercise log book and monitored by a trained exercise leader.

\section{Coordination training}

The coordination training was designed to improve fine and grossmotor body coordination. The program was led by an experienced exercise leader and focused on the improvement of complex movements for the whole body such as balance, eye-hand coordination, leg-arm coordination as well as spatial orientation and reaction to moving objects/persons. Different fitness equipment such as exercise balls, twist boards, fitness balls, jump ropes, exercise bands, and stability boards were used to offer a challenging and diversified program. Values and details of each training session (training duration, blood pressure, feelings) were recorded in an exercise logbook and monitored by a trained exercise leader. A detailed training plan can be obtained from the first author.

\section{Relaxation and stretching}

This group served as a control group to evaluate the potential effects of being involved in a guided group activity for 12 months as well as controlling for retest effects. Participants also met three times per week for $1 \mathrm{~h}$ each and were led by an experienced exercise leader. A program of relaxation techniques (e.g., imaginary journey, progressive muscle relaxation, massage), stretching and limbering for the whole body especially designed for older adults was offered for this group. Using different equipment such as fitness balls and rubber tubes provided program variation.

\section{NEUROIMAGING PROTOCOL}

The Flanker Task (see above) was performed during functional imaging using a 3 T MRI scanner in randomized block design (eight blocks, two blocks per condition, 30 s per block) alternating task conditions (congruent, incongruent, neutral, control; see above) with 30-s fixation as baseline condition. For functional scans, we employed a T2-weighted fast echo-planar imaging $(\mathrm{EPI})$ sequence $(\mathrm{TR}=2560 \mathrm{~ms}$, $\mathrm{TE}=58 \mathrm{~ms}, 200$ volumes per run with 44 slices per volume, in plane resolution $3 \mathrm{~mm} \times 3 \mathrm{~mm}$, slice thickness $3 \mathrm{~mm}$ ). Functional runs were co-registered to a high-resolution T1-weighted anatomical scan (MPRAGE sequence, $1 \mathrm{~mm} \times 1 \mathrm{~mm} \times 1 \mathrm{~mm}$ isotropic resolution).

\section{PROCEDURE}

Every 6 months, participants were tested in three individual sessions each lasting approximately $1-2 \mathrm{~h}$. Sessions took place before the start of the intervention ( $t 1)$, after 6 months ( $t 2)$, and after 12 months (t3). All three sessions took place within 1 week. At session one and two each participant completed a spiroergometry and a battery of motor and cognitive tests. At session three, differences in brain processing during the Flanker Task were measured using a 3T MRI scanner. All tasks were administered in a fixed order. 


\section{DATA ANALYSIS AND REDUCTION}

Performance in the Flanker Task and Visual Search Task was measured as the mean reaction time and the percentage of correct responses. Flanker and Visual Search Task performance was included if a participant reached at least 70\% of correct answers. In case of a premature response ( $\mathrm{RT}<50 \mathrm{~ms}$ ) or a response omission (>900 ms for the Flanker Task and RT > $2000 \mathrm{~ms}$ for the Visual Search Task) a trial was counted as error. Only correct responses were included in the outcome measure. Since prior studies have shown that physical activity is more strongly related to performance during task conditions requiring greater amounts of interference control (Hillman et al., 2006), we used the performance scores for the incongruent condition of the Flanker Task and of the conjunction search condition with 14 distractors of the Visual Search Task for statistical analysis.

\section{SELECTIVITY ANALYSIS}

Overall, of 91 participants at $t 1,44$ participants ( $48.35 \%$ of $t 1$ ) were analyzed (sample at $t 3$, i.e., participants who participated at $t 1, t 2$, and 13 ). Across the two experimental groups and the control group, the following attrition rates were obtained: In the cardiovascular group, of 33 participants at $t 1,17$ participants $(51.52 \%$ of $t 1)$ were analyzed and for the coordination group, of 31 participants at $t 1$, 16 (51.61\% of $t 1)$ were used for the final data analysis. Finally, for the control group of 27 participants at $t 1,11$ participants $(40.74 \%$ of $t 1$ ) were used. Drop out rates did not significantly differ between groups $[F(2,88)=0.44, p=0.649]$. Sample attrition is comparable to participation rates reported by other 12-month longitudinal studies with older adults (Pushkar et al., 2002; Rook and Sorkin, 2003; Manners et al., 2004). Sample attrition, however, may have major implications for the validity and interpretation of results. Thus, we performed selectivity analyses comparing the sample characteristics at $t 1$ with the sample used for data analyses (sample at 33 ) for age, education, IQ, health, activity index, BMI, hypertension, positive affect, and self-efficacy (Lindenberger et al., 2002). The objective of the selectivity analyses was to assess the extent of selectivity of the $t 3$ sample used for analysis $(n=44)$ relative to the $t 1$ parent sample $(n=91)$.

According to Lindenberger et al. (2002) sample selectivity was calculated as follows:

Selectivity $=\left(M_{\text {sample } t 3}-M_{\text {parent sample } t 1}\right) / \mathrm{SD}_{\text {parent sample } t 1}$

Mostly, selectivity was too small to even qualify for a small effect (Cohen, 1977). Overall, small sample selectivity was found for age $(\mathrm{ES}=0.23$, remaining participants were older $)$, health $(\mathrm{ES}=-0.23$, remaining participants were healthier), and positive affect $(\mathrm{ES}=0.31$, remaining participants were more positive). Given the size of these effects, it seems viable to conclude that findings obtained with the t3 sample may be generalized to the $t 1$ parent sample.

\section{STATISTICAL ANALYSIS \\ Behavioral data}

To investigate effects of the intervention programs on cardiovascular fitness repeated-measure ANOVA were computed with test SESSION $(t 1, t 2, t 3)$ as the within-subject variable and GROUP (cardiovascular training, coordination training, control group) as the between-subject variable. Effects on motor fitness were analyzed by a 3 (test SESSION: $t 1, t 2, t 3) \times 4$ (motor fitness TEST: one-leg-stand with eyes open, one-leg-stand with eyes closed, reaction speed, action speed) $\times 3$ (GROUP: cardiovascular training, coordination training, control group) repeated-measure ANOVA. To investigate effects of the intervention programs on cognitive performance repeated-measure ANOVA were computed with test SESSION $(t 1, t 2, t 3)$ and cognitive TEST (Flanker Task (incongruent condition), Visual Search Task (conjunction search condition with 14 distractors)) as within-subject variables and group (cardiovascular training, coordination training, control group) as the between-subject variable. Analyses were performed separately for performance speed and accuracy. Greenhouse Geisser adjustment is reported in case the sphericity assumption was violated. To determine effects within the exercise groups analyses were computed separately for each exercise group using estimated marginal means collapsed over the between and within-subject variables. Experimental and control groups were statistically similar to one another on measures of age, years of formal education, IQ, health, activity index, BMI, hypertension, estrogen replacement therapy (for women), and positive affect (cf. Table 1), and did not reveal noticeable selectivity effect. Thus, we abstained from including covariates.

\section{fMRI data}

Using Brain Voyager (Brain Innovation B.V., Netherlands), fMRI data were corrected for motion artifacts, temporally and spatially smoothed, and normalized to Talairach space after co-registration with the anatomical scans. General linear model (GLM) analysis was performed with two different types of regressors. For analysis of the main effect Flanker Task versus baseline (i.e., fixation) one regressor was defined using a boxcar function convolved with a canonical hemodynamic response function (HRF) combining all four flanker conditions. Further analyses were then restricted to those brain voxels that revealed significant (based on Bonferroni corrected $p$-values with $p<0.05)$ main effects of Flanker Task (combined flanker conditions) versus baseline. For the analysis of detailed effects of flanker conditions, four independent regressors ("control," "neutral," "congruent," and "incongruent") were defined. Beta values resulting from the GLM were forwarded to second level random effects analysis. There was no significant CONDITION $\times$ SESSION or CONDITION $\times$ SESSION $\times$ GROUP interaction effect. We assumed the incongruent condition to be the most sensitive one for fitness and training effects. Thus and according to the analysis of the behavioral data we based our analysis on the incongruent blocks, only. We particularly abstained from analyzing the contrast of congruent versus incongruent conditions as the so-called incongruency effect can be hardly found in blockdesigned experiments ("Gratton effect;" Gratton et al., 1992).

Those voxels were identified that revealed for the incongruent condition a significant GROUP $\times$ test SESSION interaction effect $(p<0.01$; corrected for multiple comparisons by cluster threshold estimation based on Monte Carlo simulations (Forman et al., 1995)). Estimated threshold was seven voxels. Adjacent significant voxels were defined as belonging to the same functional cluster and the anatomical regions of interest (ROI) were defined according to the Talairach coordinates of the peaks of these voxel clusters. For 
the peaks of these ROIs also the beta values for the incongruent conditions were extracted and post hoc paired-samples $t$-tests were calculated pairwise for the three sessions and for each intervention independently.

Hierarchical regression analyses were used to determine predictors of neurophysiological changes within the intervention period of 12 months. Analyses were controlled for age, education, IQ at $t 1$, health at $t 1$, activity index at $t 1$, hypertension, and estrogen replacement therapy.

\section{RESULTS \\ BEHAVIORAL RESULTS \\ Cardiovascular fitness}

For cardiovascular fitness, we found a significant SESSION by GROUP interaction $\left[F(4,41)=2.79, p=0.031, \eta^{2}=0.12\right]$, a marginally significant effect for GROUP $\left[F(2,41)=0.19, p=0.083, \eta^{2}=0.01\right]$, but no significant main effect for SESSION $[F(2,41)=1.54, p=0.220]$. After the 12-month intervention period, only older adults in the cardiovascular group demonstrated a significant overall increase of $15.7 \%$ in cardiovascular fitness $\left[F(2,40)=5.16, p=0.010, \eta^{2}=0.21\right]$, as indexed by maximal $\mathrm{VO}_{2}$ uptake but not in the coordination $(6.4 \%$ increase) and control groups (9.2\% decrease).

\section{Motor fitness}

For motor fitness, repeated-measure ANOVA revealed a significant effect of SESSION $\left[F(1.60,65.55)=37.05, p<0.001, \eta^{2}=0.48\right]$, and TASK $\left[F(1.62,66.21)=1179.04, p<0.001, \eta^{2}=0.97\right]$, and $a$ significant SESSION $\times$ TASK interaction $[F(2.98,122.31)=280.28$, $\left.p=0.001, \eta^{2}=0.13\right]$. There was no GROUP effect $[F(2,41)=0.15$, $p=0.862]$, no GROUP $\times$ SESSION interaction $[F(3.20,65.55)=1.08$, $p=0.365]$, no GROUP $\times$ TASK interaction $[F(3.23,66.21)=0.71$, $p=0.562]$ and no GROUP $\times$ SESSION $\times$ TASK interaction $[F(5.97$, $122.31)=1.42, p=0.212]$. Analysis separated by exercise groups revealed a different development for motor fitness with regard to the training program: The cardiovascular group participants revealed an improvement in feet tapping of 36.9\% $[F(2,40)=11.46$, $\left.p<0.001, \eta^{2}=0.36\right]$ and one-leg-stand with eyes closed of $52.6 \%$ $\left[F(2,40)=4.10, p=0.024, \eta^{2}=0.17\right]$. Similarly, participants of the coordination group significantly improved motor performance in feet tapping by $20.5 \%\left[F(2,40)=4.86, p=0.013, \eta^{2}=0.20\right]$, in one-leg-stand with eyes open $[36.1 \% ; F(2,40)=9.56, p<0.001$, $\left.\eta^{2}=0.32\right]$ and marginally significant in reaction speed $[10.82 \%$; $\left.F(2,40)=2.93, p=0.065, \eta^{2}=0.13\right]$. For the control group, a marginally significant improvement was found in feet tapping (action speed), only $\left[8.2 \% ; F(2,40)=2.76, p=0.075, \eta^{2}=0.12\right]$.

\section{Performance accuracy in the Flanker and Visual Search Task}

Overall, cardiovascular and coordination training groups improved their performance in the Flanker and Visual Search Task, while the control group demonstrated less change (cf. Figure 1). For performance accuracy, ANOVA revealed a significant main effect of SESSION $\left[F(1.49,59.78)=11.13, p<0.001, \eta^{2}=0.22\right]$ and TASK $\left[F(1,40)=18.97, p<0.001, \eta^{2}=0.32\right]$, and a significant SESSION by TASK interaction $\left[F(1.64,65.63)=4.52, p=0.020, \eta^{2}=0.10\right]$. There was no significant effect of GROUP $[F(2,40)=0.41, p=0.669]$, no GROUP $\times$ SESSION interaction $[F(2.99,59.78)=0.70, p=0.558]$, no GROUP $\times$ TASK interaction $[F(2,40)=0.07, p=0.932]$, and no SESSION $\times$ TASK $\times$ GROUP interaction $[F(3.28,65.63)=1.78$, $p=0.156]$. Follow-up analyses revealed that both, cardiovascular $\left[F(2,39)=4.87, p=0.013, \eta^{2}=0.20\right]$ and coordination training $\left[F(2,39)=4.10, p=0.024, \eta^{2}=0.17\right]$, led to a significant improvement of performance accuracy in the Flanker Task across the 12-month study interval, whereas the control condition did not. Furthermore, coordination training led to a significant $[F(2$, $\left.39)=5.51, p=0.008, \eta^{2}=0.22\right]$ improvement and cardiovascular training to a marginally significant $[F(2,39)=2.60, p=0.087$, $\left.\eta^{2}=0.12\right]$ improvement of performance accuracy in the Visual Search Task, whereas the control condition did not.

\section{Performance speed in the Flanker and Visual Search Task}

For performance speed, we found significant main effects for SESSION $\left[F(1.63,65.15)=12.47, p<0.001, \eta^{2}=0.24\right]$, GROUP $\left[F(2,40)=8.29, p=0.001, \eta^{2}=0.29\right]$, and TASK $[F(1,40)=515.43$, $\left.p<0.001, \eta^{2}=0.93\right]$ as well as significant GROUP $\times$ TASK $\left[F(2,40)=4.37, p=0.019, \eta^{2}=0.18\right]$ and SESSION $\times$ TASK $\left[F(1.48,59.17)=5.07, p=0.016, \eta^{2}=0.11\right]$ interactions. The GROUP $\times$ SESSION interaction was marginally significant $[F(3.26$, $65.15)=2.26, p=0.084, \eta^{2}=0.10$ ], indicating different performance developments from $t 1$ to $t 3$ for the three groups, while the SESSION $\times$ TASK $\times$ GROUP interaction was not significant $[F(2.96$, $59.17)=1.70, p=0.177]$. Follow-up analyses revealed only for the coordination group a significant performance improvement in the Visual Search Task from $t 1$ to $t 3[F(2,39)=11.82, p<0.001$, $\left.\eta^{2}=0.38\right]$. The cardiovascular group, however, showed a marginally significant improvement in their flanker reaction speed from $t 1$ to t2 $\left[F(1,41)=3.04, p=0.089, \eta^{2}=0.07\right]$.

\section{FMRI RESULTS}

For the incongruent condition of the Flanker Task, significant ${ }^{1}$ GROUP $\times$ SESSION $(t 1, t 2, t 3)$ interaction effects were found in a variety of frontal, temporal, and parietal cortical areas as well as in some subcortical structures $[F(2,42)>5.15, p<0.01$; cf. Table 2]. Adjacent significant voxels were defined as belonging to the same functional cluster and anatomical ROIs were defined according to the Talairach coordinates of the peaks of these clusters. Interaction effects for these ROIs revealed three patterns of activation change from $t 1$ to $t 3$ : (1) Increased activation for the control group from $t 1$ to t3 and nearly no change for the two experimental groups was found in the right MFG (BA 46), the left anterior and right posterior cingulate (BA 23, 24, and 32), the right parahippocampal gyrus (BA 30), the right superior temporal gyrus (BA 41), and the right lentiform nucleus (Figure 2 and Table 2, post hoc paired two-samples $t$-tests: $p<0.05)$. (2) Decreased activation for the cardiovascular group and at the same time increased activation for the control group from $t 1$ to $t 3$ was found in the left superior and middle frontal as well as bilaterally in the medial frontal gyrus (BA 6 and 9), the left anterior cingulate (ACC, BA 24 and 32), the left parahippocampal gyrus (BA 30 ), and the right superior and middle temporal gyrus (BA 21 and

${ }^{1}$ Post hoc ANCOVA with GROUP as between group factor and SESSION as within group factor and with the initial beta values as covariate revealed similar interaction effects for all but two ROIs. Only a trend was found with this analysis for the right inferior frontal gyrus (BA47) and the right lentiform nucleus $(p=0.059$ and $p=0.092$, respectively) 

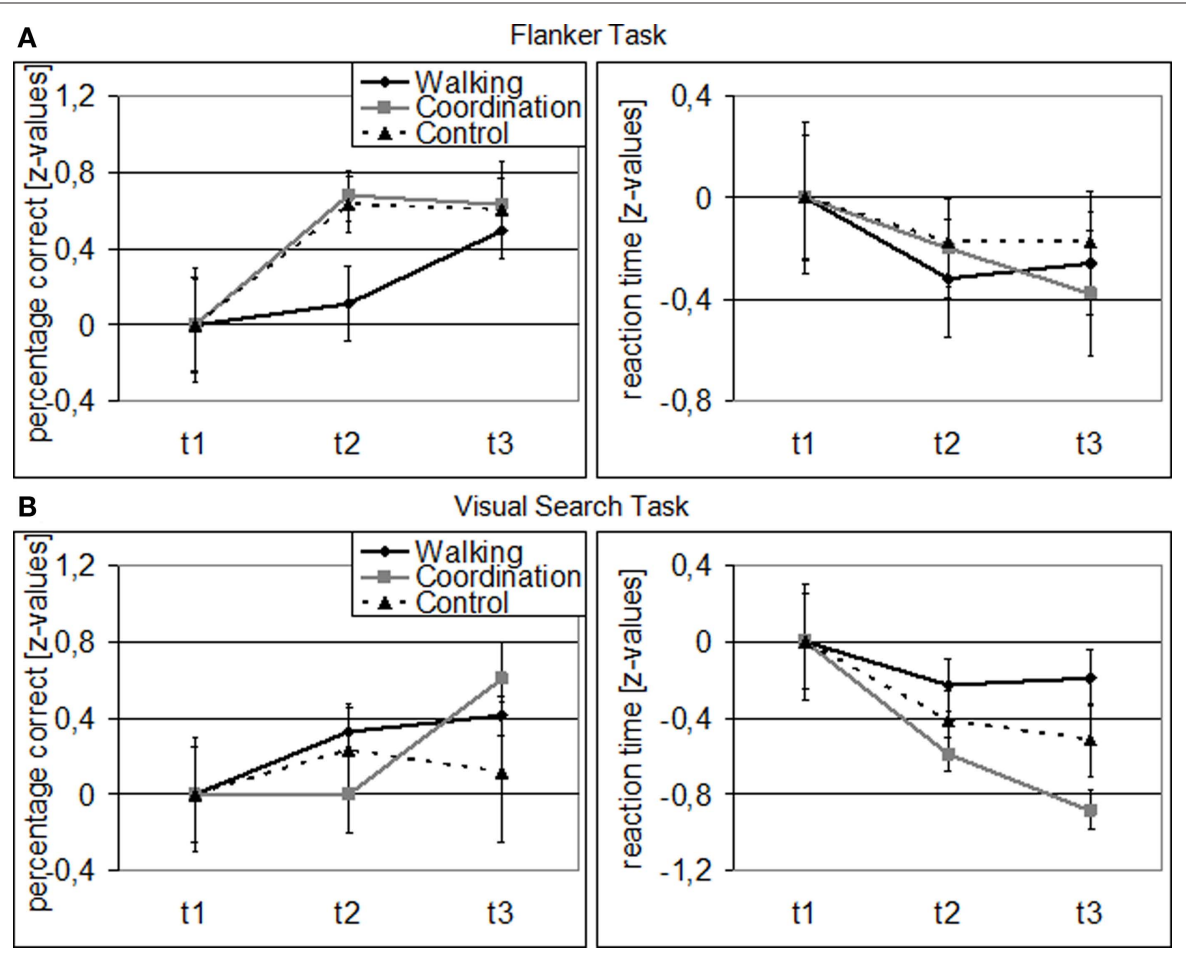

FIGURE 1 | Behavioral data for Flanker (A) and Visual Search Task (B): Z-transformed for cardiovascular training (walking), coordination training (coordination), and control group (relaxation and stretching) separately (related to $\mathbf{t}$ ). Note: Error bars represent SEM.

22). (3) For the coordination group, but not for the control group or the cardiovascular group, activation during the Flanker Task was increased after 12 months of training in the inferior frontal gyrus (bilateral BA 45 and right BA 46 and 47), thalamus and caudate, as well as in the superior parietal lobule (BA 7). As expected, the latter area revealed decreased activation for the control group (cf. Table 2).

\section{ASSOCIATION BETWEEN BEHAVIORAL AND NEUROPHYSIOLOGICAL RESULTS}

We performed hierarchical regression analyses to determine if changes in motor function predicted neurophysiological changes across the intervention period of 12 months. For this purpose, activation changes in ROIs with a significant GROUP $\times$ TEST interaction effect (cf. Table 2) were summed, as described above, to: (1) ROIs with increased activation for the control group (nearly no change for the two experimental groups), (2) ROIs with decreased activation for the cardiovascular group and at the same time increased activation for the control group, and (3) ROIs with increased activation for the coordination group. As motor variables we included change in $\mathrm{VO}_{2}$ max as indicator of cardiovascular fitness, changes in balance (one-leg-stand with eyes open) and reaction speed as indicators of coordination training, and, finally, action speed since this motor dimension revealed significant changes in the experimental and control groups. All regressions were run with difference scores of centered independent variables. Because education and hypertension correlated with motor performance, these parameters were treated as covariates in the regression analyses (step one). In step two, we analyzed the effect of changes in cardiovascular and motor fitness on neurophysiological changes.
The step 1 of the regression on the identified ROIs indicated no significant effect of hypertension or education. The step 2 of the regression analysis was significant for ROIs that showed an increased activation in the control group $\left[F(6,43)=3.84, p=0.004 ; R^{2}=0.38\right]$ and for ROIs revealing a decreased activation in the cardiovascular group together with an increased activation in the control group $[F(6,43)=2.39$, $\left.p=0.047, R^{2}=0.28\right]$. In both analysis, $\mathrm{VO}_{2}$ maxhad a significant negative explanatory power, that is, decreasing brain activation after 12 months of cardiovascular training was associated with increasing $\mathrm{O}_{2}$ supply [improved $V \mathrm{O}_{2} \max ; B=-0.70, \beta=-0.46, t(44)=-3.22, p=0.003$ ] and increasing brain activation in the control group was related to decreasing $\mathrm{O}_{2}$ supply $[B=-0.77, \beta=-0.51, t(44)=-3.83, p<0.001]$, respectively, (cf. also Table 2). The step 2 of the regression analysis for ROIs with increased activation for the coordination group did not reach significance ${ }^{2}$.

In sum, on the one hand, 12 months of cardiovascular training resulted in a reduction of task-related activation in several superior, middle and medial frontal, and superior and middle temporal cortical areas, whereas activation highly increased in these areas for the

${ }^{2}$ Detailed post hoc analysis for single ROIs that either showed an increased activation in the control group or a decreased activation in the cardiovascular group together with an increased activation in the control group also indicated that the effects seem to be mainly driven by changed $\mathrm{O}_{2}$ supply in the cardiovascular group (This is true for right and left MFG, left ACC, right posterior cingulate, right middle temporal and superior gyrus, and left parahippocampal gyrus). In detailed post hoc analysis for single ROIS with increased activation for the coordination group effects emerged only in the caudate body. Here also a positive association with motor balance was found. 
Table 2 | Regions of interest with significant group $\times$ test session interaction effects $(p<0.01)$.

\begin{tabular}{|c|c|c|c|c|c|c|c|c|c|c|c|}
\hline$x$ & $Y$ & $z$ & $\boldsymbol{F}$ & $p$ & Vox & Hem. & Area & BA & Ctl & C & $\mathbf{w}$ \\
\hline \multicolumn{12}{|c|}{$\begin{array}{l}\text { ROIS THAT SHOWED AN INCREASED ACTIVATION FOR THE CONTROL GROUP FROM } t 1 \text { TO } t 3 \text { AND (NEARLY) NO CHANGE FOR THE TWO } \\
\text { EXPERIMENTAL GROUPS }\end{array}$} \\
\hline-12 & 32 & 19 & 5.79 & 0.0060 & 31 & $\mathrm{~L}$ & Anterior cingulate & 32 & + & 0 & 0 \\
\hline 3 & -22 & 26 & 6.44 & 0.0036 & 112 & $\mathrm{R}$ & Posterior cingulate & 23 & + & 0 & 0 \\
\hline 27 & 5 & -5 & 7.40 & 0.0018 & 36 & $\mathrm{R}$ & Lentiform nucleus & l & + & $(-)$ & 0 \\
\hline \multicolumn{12}{|c|}{$\begin{array}{l}\text { ROIS THAT REVEALED A DECREASED ACTIVATION FOR THE CARDIOVASCULAR GROUP FROM } t 1 \text { TO } t 3 \text { AND AN INCREASED ACTIVATION IN } \\
\text { THE CONTROL GROUP }\end{array}$} \\
\hline-21 & 44 & 37 & 6.44 & 0.0036 & 39 & $\mathrm{~L}$ & Superior frontal & 9 & + & 0 & $(-)$ \\
\hline-35 & 5 & 46 & 7.01 & 0.0024 & 45 & $L$ & Middle frontal & 6 & + & 0 & - \\
\hline 63 & -4 & -2 & 7.84 & 0.0013 & 89 & $\mathrm{R}$ & Middle temporal & 21 & + & 0 & - \\
\hline 60 & 2 & -5 & 6.15 & 0.0045 & 32 & $\mathrm{R}$ & Superior temporal & 22 & $(+)$ & 0 & - \\
\hline \multicolumn{12}{|c|}{$\begin{array}{l}\text { ROIS THAT REVEALED AN INCREASED ACTIVATION FOR THE COORDINATION GROUP FROM } t 1 \text { TO } t 3 \text { AND NO CHANGE OR DECREASE IN } \\
\text { THE OTHER TWO GROUPS }\end{array}$} \\
\hline-33 & 26 & 7 & 9.07 & 0.0005 & 470 & $\mathrm{~L}$ & Inferior frontal & 45 & 0 & + & 0 \\
\hline 60 & 21 & 19 & 9.75 & 0.0003 & 149 & $\mathrm{R}$ & Inferior frontal & 45 & 0 & + & 0 \\
\hline 54 & 44 & 10 & 8.50 & 0.0008 & 509 & $\mathrm{R}$ & Inferior frontal & 46 & 0 & + & 0 \\
\hline 49 & 20 & -11 & 5.28 & 0.0090 & 181 & $\mathrm{R}$ & Inferior frontal & 47 & 0 & + & 0 \\
\hline 15 & -46 & 61 & 6.14 & 0.0046 & 225 & $\mathrm{R}$ & Superior parietal & 7 & - & + & 0 \\
\hline 9 & -1 & 4 & 8.92 & 0.0006 & 268 & $\mathrm{R}$ & Thalamus & I & 0 & + & 0 \\
\hline 9 & 8 & 7 & 6.32 & 0.0040 & 75 & $\mathrm{R}$ & Caudate body & l & 0 & $H$ & 0 \\
\hline
\end{tabular}

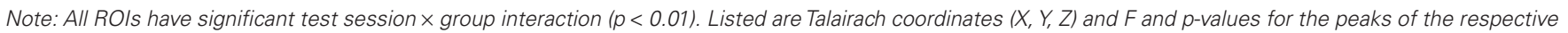

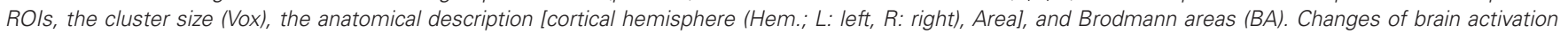

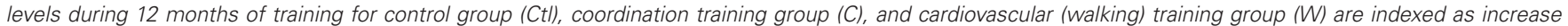

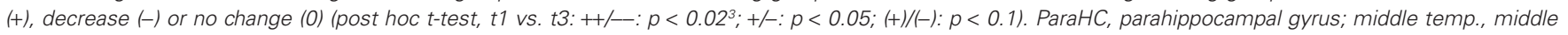
temporal gyrus.

control group. For the coordination group, on the other hand, we found increased activation after training particularly in the inferior frontal gyrus and the superior parietal cortex as well as subcortical structures like thalamus and caudate body. In both experimental groups these changes were linear in nature and showed no leveling off during the 12 months. Changes in task-related activation in the cardiovascular training and the control group seem to be mainly driven by changes (increases and decreases, respectively) in $\mathrm{VO}_{2}$ max. Increased brain activations in visual-spatial areas in the coordination group were not associated with changes in motor performance.

\section{DISCUSSION}

The aims of this study were threefold. First, we expected to replicate extant results on the facilitating effects of cardiovascular training on cognitive functioning in older adults, second we expected that two types of physical training (cardiovascular and coordination training) show differential effects on cognitive functioning in older adults, and

${ }^{3}$ Multiple testing increases the risk for chance findings due to potential alpha error accumulation. Thus, we adjusted the nominal alpha level for the three comparisons (Ctl, C, W) following Bonferroni (adjusted $\alpha=0.017$ ). third we explored whether longer training periods, that is, a period of 12 months, indicated continued performance increases or rather a leveling off of intervention effects. Results by large confirmed our expectations.

\section{REPLICATING AND SPECIFYING THE FACILITATIVE EFFECT OF CARDIOVASCULAR TRAINING}

First, our results indeed replicated earlier studies on the effect of cardiovascular training on cognitive functioning in older adults as we confirmed that 6 months of cardiovascular training resulted in better performance and more efficient brain functioning when working on executive control tasks. Similar to Colcombe et al. (2004), we showed an increase in cardiovascular fitness after 6 months of walking training using a modified version of the Erickson Flanker Task. We also found a reduced level of activity in the ACC, a region associated with the experience of behavioral conflict and the need to adapt attentional control processes; however, our fMRI results in certain respects also diverged from the findings of Colcombe et al. (2004). Where they had found significantly higher task-related activity in attentional control areas (MFG, SFG, SPL) after the 6-month training period, we found reduced activity in these areas as compared to controls (cf. Table 2). 

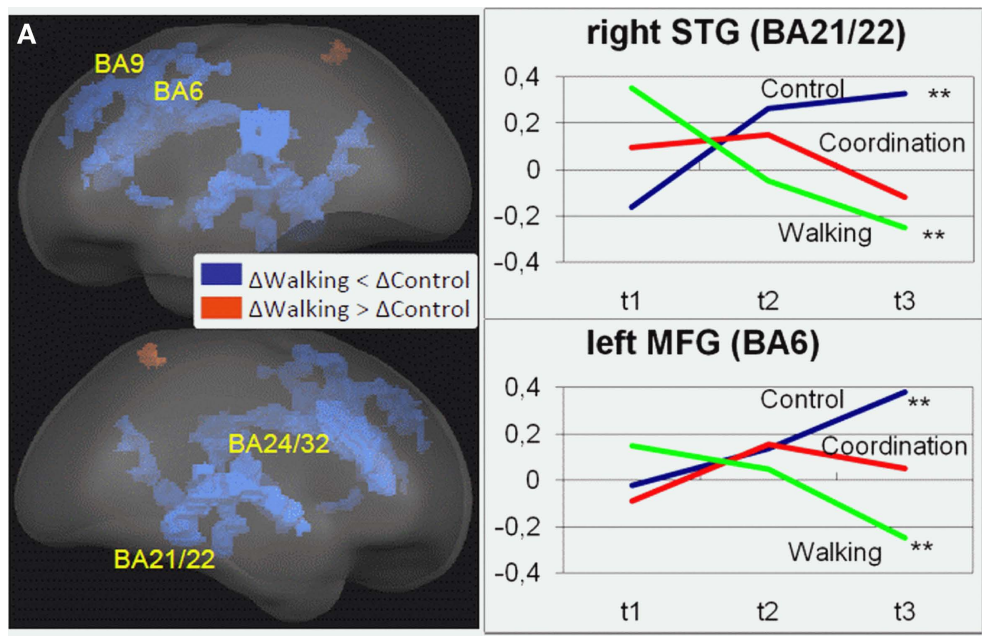

B

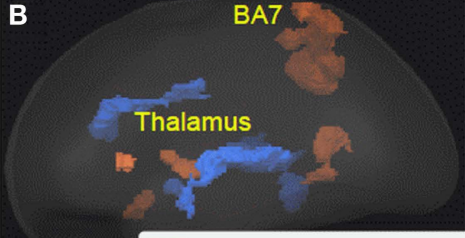

$\Delta$ Coordination $<\Delta$ Control $\Delta$ Coordination $>\Delta$ Control 43 BA41/22 right STG (BA41/22)

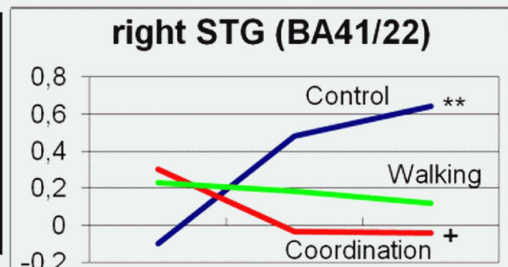

$-0,2$

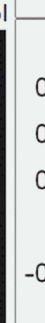

t1 t2

right IFG (BA47)

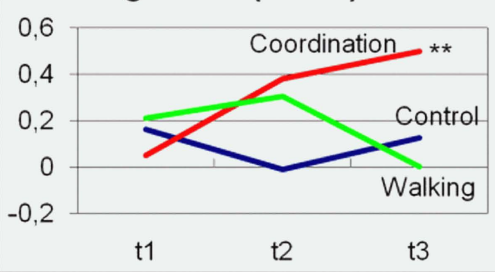

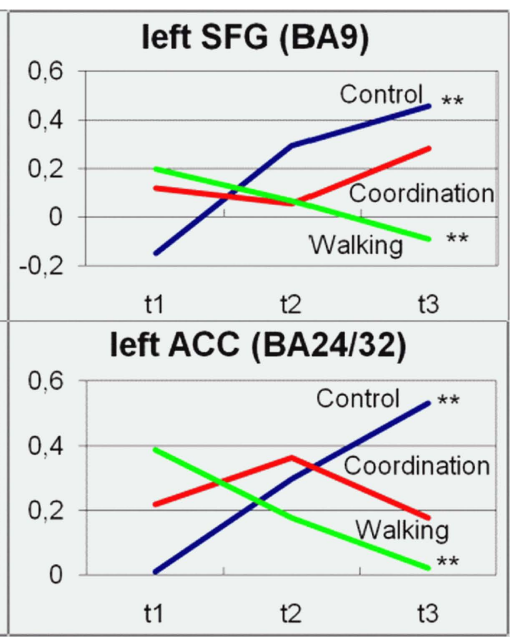

right Thalamus

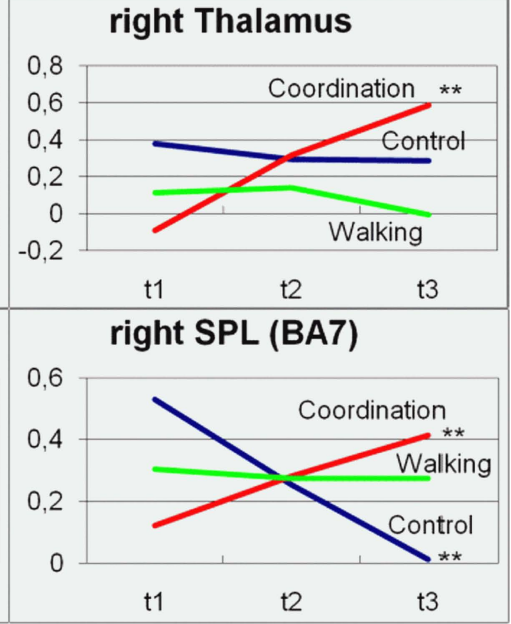

FIGURE 2 | Regions of interest (ROIs) that revealed significant contrasts for (A) cardiovascular training (walking) ( $t 3-t 1)>$ control group (control) ( $t 3-t 1$ ) and $(B)$ coordination training group (coordination) $(t 3-t 1)>$ control group (control) $(t 3-t 1)$ are projected into a standardized glass brain normalized to
Talairach space. Note: For (A) and (B) representative ROls are indicated as Brodmann areas (see Table 2 for cluster descriptions) and for these ROls changes in beta estimates from $t 1$ to $t 3$ are shown on the right for the three experimental groups with ${ }^{*} p<0.02,{ }^{*} p<0.05,+p<0.1$ (cf. Table 2)
Our results support Hollmann et al. (2007) findings that older adults showed similar cognitive performance and brain activation patterns as younger adults after cardiovascular training. Their activation patterns differed significantly from that of untrained older adults. The untrained older adults showed lower cognitive performance accompanied by an activation of larger brain areas, whereas the trained participants showed less activation and cognitive performance levels comparable to that of younger adults. Hollmann et al. (2007) concluded that the brain exhibited a status of more efficient processing as a result of physical training, similar to the cardiovascular adaptations observed after training.

\section{DIFFERENTIAL EFFECTS OF CARDIOVASCULAR AND COORDINATION TRAINING ON COGNITIVE FUNCTIONING}

Second, we showed, for the first time, that a different form of physical training other than cardiovascular, coordination training, also improved cognitive functioning in older adults. And that brain activation patterns associated with exercise-related performance changes differed between different types of physical training. We found an increase in Flanker Task performance accuracy and in Visual Search Task performance accuracy and speed.

The control group showed no significant performance increases; however, their neurophysiological data revealed increased taskrelated activations in superior, middle, and medial frontal, as well as superior and middle temporal cortical areas, whereas the coordination group revealed unchanged activation patterns, albeit performance increases. This may reflect the fact that less compensatory activation is needed to perform the Flanker Task for older adults who underwent coordination training. In light of the aging literature (e.g., Park and Reuter-Lorenz, 2009), stable performance accuracy of the control group associated with additional frontal activation may be interpreted as the ability to recruit additional frontal resources for compensatory purposes.

Furthermore, the coordination group activation level during the Flanker Task was greater after 6 and 12 months of training particularly in the right inferior frontal gyrus and the superior 
parietal cortex, which form part of the so-called visuo-spatial attention network (Corbetta and Shulman, 2002; De Graaf et al., 2009), as well as the thalamus and caudate body. These latter subcortical structures are more important for process automation without conscious control (Aron et al., 2003). This activation increase might contradict the beneficial reduction of prefrontal activations described above. However, it has been suggested that increased prefrontal activation is the consequence of age-related structural and functional decline in various brain regions. That is, increased prefrontal activation might compensate for processing impairment, particularly in posterior areas, by stronger efforts in executive control (Greenwood, 2007). Similarly, less prefrontal activation or no increase in activation after cardiovascular training indicates a reduced need for compensation or increased cognitive control, whereas coordination training seems to specifically (re-)strengthen parts of the networks that are particularly impaired during aging leading to more automated motor responses and more effective processing and integration of visuo-spatial information. Thus, the differential relation of cardiovascular and coordination training with changes in brain activation for the cognitive task might indicate that the brain processes underlying the performance changes differ depending on the intervention.

This assumption is supported by results of the cross-sectional analysis indicating a differential association of physical and motor fitness with cognitive functioning on the behavioral and neurophysiological level (Flegal and Reuter-Lorenz, 2010; Voelcker-Rehage et al., 2010). Both, physical and motor fitness, were related to more efficient cognitive processing, indicated by less cortical activations in the superior and middle frontal cortex, whereas motor fitness was also related to higher activation of the right inferior frontalposterior parietal network. Also results of the visual search task support the assumption of improved processing and integration of visuo-spatial information. Although, we have only behavioral data available, these regions have been shown to be also involved in visual conjunction search (Mueller et al., 2003).

Furthermore, results of the spiroergometry confirmed that cardiovascular training mainly improved cardiovascular performance whereas coordination training and relaxation and stretching (control group) did not (significant test session by group interaction). Inversely, coordination training mainly facilitated motor fitness, that is, balance and reaction speed ${ }^{4}$. Results of the regression analysis support the assumption that changes in physical fitness predict neurophysiological changes. As shown in Table 2, fMRI analysis revealed (1) ROIs with increased activation for the control group (nearly no change for the two experimental groups), (2) ROIs with decreased activation for the cardiovascular group and at the same time increased activation for the control group, and (3) ROIs with increased activation for the coordination group (no change for cardiovascular and control group). Pre- to post-test changes in beta estimates that revealed increased activation for the control group were associated with reductions in $\mathrm{VO}_{2}$ max. Increased activation

${ }^{4}$ Interestingly, cardiovascular training not only improved $\mathrm{VO}_{2} \max$, but also balance performance in one test (one-leg-stand with eyes closed). Effect of walking on postural stability has also been shown by others (Melzer et al., 2003). When analyses were controlled for performance increases in balance, effects of cardiovascular training on cognitive performance, however, remained unchanged. came along with decreased $\mathrm{VO}_{2} \max \left(\mathrm{VO}_{2}\right.$ max decreased in the control group by 9.2\%). Conversely, decreased activation of the cardiovascular group (and at the same time increased activation for the control group in these areas) was associated with increases in $\mathrm{VO}_{2}$ max. Interestingly, changes induced by coordination training had only low predictive power for neurophysiological changes. It is not clear whether this reflects less specific association between behavioral and neurophysiological changes for the coordination training group. Another alternative is that the changes in the motor system due to coordination training were not adequately measured by the selected motor tests.

Animal studies have shown that cardiovascular and coordination training effect brain processes differently. Rats regularly running on a treadmill (cardiovascular training) had an increase in the density of capillaries (Black et al., 1990) and shorter diffusion distance from blood vessels in the molecular layer of the paramedian lobule (Isaacs et al., 1992) without an increase in the synaptic numbers. Rats that participated in motor-skill learning tasks (e.g., traverse an elevated obstacle course requiring substantial motor coordination to complete; comparable to coordination training), however, showed a greater number of synapses per neuron without an increase in the density of capillaries. Rats that were taught complex motor skills substantially increased the volume of the molecular layer per Purkinje neuron and sufficiently increased the number of blood vessels to maintain the diffusion distance. Furthermore, they had significantly more parallel fiber to Purkinje cell synapses than walking and inactive animals (e.g., Isaacs et al., 1992). Obviously, we do not have available further physiological data that would allow investigating the mediating mechanisms, but based on the animal studies one can speculate that the observed decrease in frontal activation in the cardiovascular group is facilitated by a better $\mathrm{O}_{2}$ supply, caused by a higher density of capillaries and/or shorter diffusion distances for $\mathrm{O}_{2}$. Whereas, coordination training requires perceptual and higher-level cognitive processes, such as attention, that are essential for mapping sensation to action and ensuring anticipatory and adaptive aspects of postural control or coordination. Thus, coordination training might mainly facilitate the handling of visual and spatial information (Smith and Baltes, 1999) by increasing the number of synapses per neuron in the demanded areas. As the fMRI signal is mainly based on synaptic activity (Logothetis, 2008), this might be expressed by a higher activation of afore mentioned areas as shown by the fMRI data.

\section{THE EFFECT OF LONGER TRAINING PERIODS (12 MONTHS) ON COGNITIVE FUNCTIONING}

Third, we showed that not only 6 months of cardiovascular training had beneficial effects on cognitive functioning (on behavioral and neurophysiologic level), but that a specific pattern of training gains continued during the 12-month period. In that regard, our results extend existing findings. To the best of our knowledge, no study exists that has demonstrated changes on the behavioral as well as the neurophysiological level across such a long test period. Based on the beta estimates (cf. Figure 2) alterations in brain activation patterns impressively revealed that benefits from gains in cardiovascular fitness did not reach a ceiling after 6 months of training, but continued thereafter without being fully associated with behavioral effects. Also, during the second 6 months, changes 
in brain activation (increases and decreases) were observed for most of the described areas. This is particularly true for the middle and superior frontal regions for the control and the cardiovascular group and for the inferior frontal cortex for the coordination group. This finding seems to indicate that the potential of the aging brain to increase efficiency based on physical training is not easily exhausted and one could infer that an even longer training program might further improve cognitive functioning also on the behavioral level.

While the neurophysiological effects were pervasive, on the behavioral level effects were weaker. It may be the case that the behavioral indicators used were not quite sensitive enough to reflect such continued changes in brain activation patterns or that the continued neurophysiological change is associated with different behavioral indicators than speed and accuracy. Given the relatively small sample size and the high variability within the intervention groups, we may also consider the possibility that there was not enough statistical power for the continued behavioral changes to gain significance. Similarly, Williamson et al. (2009) found no strong group differences in cognitive performance on the behavioral level across 12 months. We infer from this that there is no 1:1 relationship between neurophysiological and cognitive changes. Rather, it is speculated that neurophysiological changes only become observable on a behavioral level when a certain threshold of structural/ functional changes (i.e., changes in functional integration) has been surpassed (Cabeza, 2001). This means that positive effects of physical training can be expressed in changes of brain activity, however, not necessarily do they have to simultaneously manifest themselves in changes of cognitive performance.

A further limitation of our study is that only the Flanker Task was performed within the MRI machine. The reason was that inhibitory processes are thought to play a central role in age-related losses in

\section{REFERENCES}

Ainsworth, B. E., Haskell, W. L., Whitt, M. C., Irwin, M. L., Swartz, A. M., Strath, S. J., O’Brien, W. L., Bassett, D. R. Jr., Schmitz, K. H., Emplaincourt, P. O., Jacobs, D. R. Jr., and Leon, A. S. (2000). Compendium of physical activities: an update of activity codes and MET intensities. Med. Sci. Sports Exerc. 32, S498-S516.

Anderson, B. J., Alcantara, A. A., Isaacs, K. R., Black, J. E., and Greenough, W. T. (1994). Glial hypertrophy is associated with synaptogenesis following motorskill learning, but not with angionesis following exercise. Glia 11, 73-80.

Aron, A. R., Schlaghecken, F., Fletcher, P. C., Bullmore, E. T., Eimer, M., Barker, R., Sahakian, B. J., and Robbins, T. W. (2003). Inhibition of subliminally primed responses is mediated by the caudate and thalamus: evidence from functional MRI and Huntington's disease. Brain 126, 713-723.

Bach, M. (2007). The Freiburg Visual Acuity Test - variability unchanged by post-hoc re-analysis. Graefes Arch. Clin. Exp. Ophthalmol. 245, 965-971.

Bäckman, L., Lindenberger, U., Li, S.-C., and Nyberg, L. (2010). Linking

many cognitive functions. As shown by the results of the Visual Search Task and by earlier cross-sectional analyses (VoelckerRehage et al., 2010), however, also other cognitive tasks seem to be related particularly to coordination training or motor fitness, respectively.

\section{CONCLUSION}

Studies of physical fitness effects on cognitive function have typically focused on cardiovascular training over short intervention periods and neglected additional effects of physical activity. Extending earlier results about the positive effects of cardiovascular fitness and exercise on general cortical processing efficiency, our study revealed that even coordination training has effects on specific brain networks. Moreover, our longitudinal data confirm recent cross-sectional results showing that physical and motor fitness differentially relate to cognitive processes (Voelcker-Rehage et al., 2010). A more precise understanding of the cognitive benefits of various types of physical training can guide individuals and training professionals with regard to efficient training selection.

\section{ACKNOWLEDGMENTS}

This project was supported by a grant from the Robert Bosch Foundation (12.5.1366.0005.0) and from the German health insurance company DAK as well as funds of the Jacobs Center on Lifelong Learning and Institutional Development. We would like to thank Astrid Beck, Mala Kamenidu, Alexander Kowanz, Kathrin Linke, Fabian May, Pit Pletz, Tanja Truhart, and Julia Wolf for conducting the training interventions, Laura Fettkenhauer, Alexander Knop, Fabian May, and Pit Pletz for their help with data collection, Melanie Löbe and Peter Erhardt for their help with conducting the MRI scans, and all participants for participating in the study.

Corbetta, M., and Shulman, G. L. (2002) Control of goal-directed and stimulusdriven attention in the brain. Nat. Rev. Neurosci. 3, 201-215.

De Graaf, T.A., Roebroeck, A., Goebel, R., and Sack, A. T. (2009). Brain network dynamics underlying visuospatial judgment: an fMRI connectivity study. J. Cogn. Neurosci. 22, 2012-2026.

Ekdahl, C., Jarnlo, G. B., and Andersson, S. I. (1989). Standing balance in healthy subjects. Scand. J. Rehabil. Med. 21, 187-195.

Ehrler, W., and Huth, M. (2000). "Körperliche Leistungsfähigkeit 43bis 45-Jähriger und 62- bis 64-Jähriger im Vergleich [A comparison of the motor performance of 43-45 and 62-64 year olds]," in Aspekte der Entwicklung im mittleren und höheren Lebensalter, eds P. Martin, K. U. Ettrich, U. Lehr, D. Roether, and A. FischerCyrulies (Darmstadt: Steinkopff), 220-234.

Flegal, K. E., and Reuter-Lorenz, P. A. (2010). Commentary aging and brain fitness. Eur. J. Neurosci. 31, 165-166.

Folstein, M. F., Folstein, S. E., and McHugh, P. R. (1975). "Mini-mental state”. A practical method for grad- ing the cognitive state of patients for the clinician. J. Psychiatr. Res. 12, 189-198.

Forman, S. D., Cohen, J. D., Fitzgerald, M., Eddy, W. F., Mintun, M. A., and Noll, D. C. (1995). Improved assessment of significant activation in functional magnetic resonance imaging ( $\mathrm{fMRI}$ ): use of a cluster-size threshold. Magn. Reson. Med. 33, 636-647.

Geurts, A., Knoop, J. A., and van Limbeck, J. (1999). Is postural control associated with mental functioning in the persistent postconcussion syndrome? Arch. Phys. Med. Rehabil. 80, 144-149.

Gratton, G., Coles, M. G., and Donchin, E. (1992). Optimizing the use of information - strategic control of activation of responses. J. Exp. Psychol. Gen. 121, 480-506.

Greenwood, P. M. (2007). Functional plasticity in cognitive aging: review and hypothesis. Neuropsychology 21, 657-673.

Hillman, C. H., Motl, R. W., Pontifex, M. B., Posthuma, D., Stubbe, J. H., Boomsma, D. I., and de Geus, E. J. C. (2006). Physical activity and cognitive function in a cross-section of younger 
and older community-dwelling individuals. Health Psychol. 25, 678-687.

Hollmann, W., Strüder, H. K., Tagarakis, C.V.M., and King, G. (2007). Physical activity and the elderly. Eur. J. Cardiovasc. Prev. Rehabil. 14,730-739.

Hommel, B., Li, K. Z. H., and Li, S. C. (2004). Visual search across the life span. Dev. Psychol. 40, 545-558.

Isaacs, K. R., Anderson, B. J., Alcantara, A. A., Black, J. E., and Greenough, W. T. (1992). Exercise and the brain: angionesis in the adult rat cerebellum after vigorous physical activity and motor skill learning. J. Cereb. Blood Flow Metab. 12, 110-119.

Kessler, E. M., and Staudinger, U. M. (2009). Affective experience in adulthood and old age: the role of affective arousal and perceived affect regulation. Psychol. Aging 24, 349-362.

Kramer, A. F., and Erickson, K. I. (2007). Capitalizing on cortical plasticity: influence of physical activity on cognition and brain function. Trends $\operatorname{Cog} n$. Sci. 11, 342-348.

Li, S. C., Lindenberger, U., Hommel, B., Aschersleben, G., Prinz, W., and Baltes, P. B. (2004). Transformations in the couplings among intellectual abilities and constituent cognitive processes across the lifespan. Psychol. Sci. 15, 155-163.

Lindenberger, U., Singer, T., and Baltes, P. B. (2002). Longitudinal selectivity in aging populations: separating mortality-associated versus experimental components in the Berlin agign study. J. Gerontol. Psychol. Sci. 57B, P474-P482.

Logothetis, N. K. (2008). What we can do and what we cannot do with fMRI. Nature 453, 869-878.

Lövdén, M., Bäckman, L., Lindenberger, U., Schäfer, S., and Schmiedek, F. (2010). A theoretical framework for the study of adult cognitive plasticity. Psychol. Bull. 136, 659-676.

Lustig, C., Shah, P., Seidler, R., and ReuterLorenz, P. A. (2009). Aging, training, and the brain: a review and future directions. Neuropsychol. Rev. 19, 504-522.

Manners, J., Durkin, K., and Nesdale, A. (2004). Promoting advanced ego development among adults. J. Adult. Dev. 11, 19-27.

Melzer, I., Benjuya, N., and Kaplanski, J. (2003). Effects of regular walking on postural stability in the elderly. Gerontology 49, 240-245.

Meyer, T., Lucia, A., Earnest, C. P., and Kindermann, W. (2005). A conceptual framework for performance diagnosis and training prescription from submaximal gas exchange parameters theory and application. Int. J. Sports Med. 26(Suppl. 1), S38-S48.

Mueller, N. G., Donner, T. H., Bartelt, O. A., Brandt, S. A., Villringer, A., and Kleinschmidt, A. (2003). The functional neuroanatomy of visual conjunction search: a parametric fMRI study. Neuroimage 20, 1578-1590.

Park, D. C., and Reuter-Lorenz, P. A. (2009). The adaptive brain: aging and neurocognitive scaffolding. Annu. Rev. Psychol. 60, 173-196.

Planinsec, J. (2002). Relations between the motor and cognitive dimension of preschool girls and boys. Percept. Mot. Skills 94, 415-423.

Porszasz, J., Casaburi, R., Somfay, A., Woodhouse, L. J., and Whipp, B. J. (2003). A treadmill ramp protocol using simultaneous changes in speed and grade. Med. Sci. Sports Exerc. 35, 1596-1603.

Prioux, J., Ramonatxo, M., Hayot, M., Mucci, P., and Prefaut, C. (2000). Effect of ageing on the ventilatory response and lactate kinetics during incremen- tal exercise in man. Eur. J.Appl. Physiol. 81, 100-107.

Pushkar, D., Reis, M., and Morros, M. (2002). Motivation, personality, and well-being in older volunteers. Int. J. Aging Hum. Dev. 55, 141-162.

Rikli, R. E., and Edwards, D. (1991). Effects of a three-year exercise program on motor function and cognitive processing speed in older women. Res. Q. Exerc. Sport 62, 61-67.

Rook, K. S., and Sorkin, D. H. (2003). Fostering social ties through a volunteer role: implications for older-adults' psychological health. Int. J. Aging Hum. Dev. 57, 313-337.

Smiley-Oyen, A. L., Lowry, K. A., Francois, S. J., Kohut, M. L., and Ekkekakis, P. (2008). Exercise, fitness, and neurocognitive function in older adults: the "selective improvement" and "cardiovascular fitness" hypotheses. Ann. Behav. Med. 36, 280-291.

Smith, J., and Baltes, P. B. (1999). “Trends and profils of psychological functioning in very old age," in The Berlin Aging Study, eds P. B. Baltes and K. U. Mayer (New York: Cambridge University Press), 197-226.

Uhrich, T. A., and Swalm, R. L. (2007). A pilot study of a possible effect from a motor task on reading performance. Percept. Mot. Skills 104, 1035-1041.

van Praag, H., Shubert, T., Zhao, C., and Gage, F. H. (2005). Exercise enhances learning and hippocampal neurogenesis in aged mice. J. Neurosci. 25, 8680-8685.

Voelcker-Rehage, C., Godde, B., and Staudinger, U. M. (2010). Cardiovascular and motor fitness are both related to cognition in old age. Eur. J. Neurosci. 31, 167-176.

Voelcker-Rehage, C., and Wiertz, O. (2003). Die Lernfähigkeit sportmotorischer Fertigkeiten im Lichte der Entwicklungspsychologie der Lebensspanne [Motor Skill Learning in Focus of Lifespan Developmental Psychology]. Bielefeld: Universität Bielefeld.

Williamson, J. D., Espeland, M., Kritchevsky, S. B., Newman, A. B., King, A. C., Pahor, M., Guralnik, J.M., Pruitt, L.A., and Miller,M.E. (2009). Changes in cognitive function in a randomized trial of physical activity: results of the lifestyle interventions and independence for elders pilot study. J. Gerontol. A Biol. Sci. Med. Sci. 64A, 688-694.

Yund, E. W. (2003). Simultaneous auditory thresholds at multiple frequencies and for both ears. Arch. Neurobehav. Exp. Stimuli 69. Available at: http://www.neurobs.com/ex_files/ expt_view?id $=69$

Conflict of Interest Statement: The authors declare that the research was conducted in the absence of any commercial or financial relationships that could be construed as a potential conflict of interest.

Received: 04 October 2010; accepted: 02 March 2011; published online: 17 March 2011.

Citation: Voelcker-Rehage C, Godde B and Staudinger UM (2011) Cardiovascular and coordination training differentially improve cognitive performance and neural processing in older adults. Front. Hum. Neurosci. 5:26. doi: 10.3389/ fnhum.2011.00026

Copyright $\odot 2011$ Voelcker-Rehage, Godde and Staudinger. This is an open-access article subject to an exclusive license agreement between the authors and Frontiers Medio $S A$, which permits unrestricted use, distribution, and reproduction in any medium, provided the original authors and source are credited. 